\title{
Searches for 4th generation and vector-like quarks with the ATLAS detector
}

\author{
Lea Gauthier* \\ CEA-Saclay, IRFU/SPP - Université de Montreal \\ On behalf of the ATLAS Collaboration \\ E-mail: lea.gauthierecern.ch
}

\begin{abstract}
Fourth Generation Quarks and Vector Like Quarks (VLQ) have been searched for in ATLAS using proton-proton collisions at $\sqrt{s}=7 \mathrm{TeV}$ with integrated luminosity between $1.04 \mathrm{fb}^{-1}$ and $4.7 \mathrm{fb}^{-1}$. Single lepton as well as opposite sign and same-sign dilepton signatures have been analysed to search for $b^{\prime}, t^{\prime}$ or exotic particles with charge $Q=-1 / 3,2 / 3$ and $5 / 3$. The data were found consistent with the expected background from Standard Model processes and limits have been extracted. Based on a model of fourth generation quarks, the following bounds were obtained at $95 \%$ confidence level $(\mathrm{CL}): \mathrm{m}(Q)>350 \mathrm{GeV}$ for up and down type quark $Q, \mathrm{~m}\left(t^{\prime}\right)>656 \mathrm{GeV}$ for $t^{\prime} \rightarrow W+b$ and $\mathrm{m}\left(b^{\prime}\right)>670 \mathrm{GeV}$ for $b^{\prime} \rightarrow W+t$. A Vector Like Quark (VLQ) interpretation of $t^{\prime}$ yields upper limits in the two-dimensional plane of $B R\left(t^{\prime} \rightarrow W b\right)$ versus $B R\left(t^{\prime} \rightarrow H t\right)$ as a function of the $t^{\prime}$ mass. A vector-like singlet $b^{\prime}$ (with $b^{\prime} \rightarrow Z+b$ ), mixing solely with the third SM generation is excluded for $\mathrm{m}\left(b^{\prime}\right)<358 \mathrm{GeV}$. Limits on VLQs with charge $5 / 3$ has been obtained for different coupling values $\lambda\left(T_{5 / 3}-t-W\right)$ giving $m\left(T_{5 / 3}\right)>670 \mathrm{GeV}$ for $\lambda \ll 1, \mathrm{~m}\left(T_{5 / 3}\right)>$ $680 \mathrm{GeV}$ for $\lambda=1$ and $\mathrm{m}\left(T_{5 / 3}\right)>700 \mathrm{GeV}$ for $\lambda=3$. Single production of VLQs coupling to light generations have also been investigated for VLQs with different charges with the results $\mathrm{m}\left(Q_{-1 / 3}\right)>1.08 \mathrm{TeV}, \mathrm{m}\left(Q_{2 / 3}\right)>1.12 \mathrm{TeV}$ and $\mathrm{m}\left(Q_{5 / 3}\right)>1.42 \mathrm{TeV}$.
\end{abstract}

Xth Quark Confinement and the Hadron Spectrum,

October 8-12, 2012

TUM Campus Garching, Munich, Germany

\footnotetext{
* Speaker.
} 


\section{Introduction}

The Standard Model (SM) of particle physics must be incomplete since a low Higgs mass requires unnatural fine tuning and since it leaves many experimental observations unexplained, such as the matter-antimatter asymmetry in the universe. Two particular models are here studied that might solve some of these problems : models with a fourth generation of heavy chiral fermions $\left(t^{\prime}, b^{\prime}\right)$ provide new sources of $\mathrm{CP}$ violation and allow for a heavier Higgs boson. Vector-like quarks appear in many theories beyond the Standard model, as they solve the hierarchy of energy scale and, in some models, explain the FB asymmetry observed in $t \bar{t}$ production.

\subsection{Fourth Generation Quarks Model}

A fourth generation of heavy chiral quarks is a natural extension to the SM. This model is based on a new chiral generation : a $S U(2)_{L} \operatorname{doublet}\left(t^{\prime}, b^{\prime}\right)_{L}$ with the corresponding right-handed singlets $t_{R}^{\prime}, b_{R}^{\prime}$. It can provide a new source of CP violation in $B_{S}$ decays to explain the matter-antimatter asymmetry in the Universe and allows for a heavier Higgs boson, while remaining consistent with precision electroweak measurements [1] if one assumes small mass splitting between the heavy up-type quark $\left(t^{\prime}\right)$ and the heavy down-type quark $\left(b^{\prime}\right)$. This model is disfavoured, however, by $m_{H} \sim 126 \mathrm{GeV}[2]$.

\subsection{Vector Like Quarks Model}

Vector-like quarks, defined as quarks for which both chiralities have the same transformation properties under the electroweak group $S U(2) \times U(1)$ are predicted by many extensions of the $\mathrm{SM}$ (Grand Unification, extra dimensions, ..). VLQs are often introduced as a top partner to cancel the quadratic divergence of the top loop in the Higgs propagator. Their masses are not bound by vacuum stability arguments or by Higgs properties. Since the couplings of the light quarks are well constrained, if VLQs exist they are generally expected to only couple sizeably to the third generation. However, in certain scenarios, mixing to first generations is not excluded, since corrections to the quark mixings can cancel, relaxing these constraints. Details can be found in [3].

\section{Analysis}

In this document, analysis of the lepton+jets channel and dilepton channel are presented, based on datasets recorded in the ATLAS detector [4] at $\sqrt{s}=7 \mathrm{TeV}$ with integrated luminosity between $1.04 \mathrm{fb}^{-1}$ and $4.7 \mathrm{fb}^{-1}$.

Isolated leptons are selected with $p_{T} \geq 20$ (25) GeV for muons (electrons) and a pseudorapidity $|\eta| \leq 2.5$ (2.45 and $1.37 \leq|\eta| \leq 1.52$ ) for muons (electrons). The jets are reconstructed with the anti $-k_{t}$ algorithm [5] for a radius parameter of 0.4 and should have $p_{T} \geq 25 \mathrm{GeV}$ and $|\eta| \leq 2.5$. The limits presented here are all extracted using the CL method [6], except for single production of VLQ coupling to light generations which use a Bayesian approach.

\subsection{Background}

All backgrounds estimated with MC generators are normalized to the best known theoretical cross-section. 
- For the dilepton final state : dibosons ( $W W, W Z$ and $Z Z)$ are estimated with $\mathrm{MC}$ generators while fake leptons are based on a data driven method (matrix method). For the analysis based on opposite-sign leptons, $t \bar{t}$, single top, $Z+$ jets and the Drell-Yan background are taken into account. For the analysis requiring same-sign leptons, $t \bar{t} W, t \bar{t} Z, t \bar{t} W W$ and $W W+2$ jets are estimated with MC generators while the charge-flip background is estimated with a data-driven likelihood method.

- For the single lepton final state : dibosons, $t \bar{t}$, single top, $W+$ jets, $Z+$ jets, $t \bar{t} W, t \bar{t} Z, t \bar{t} W W$ and $W W+2$ jets are estimated with MC generators while the multi-jet background is estimated with a data-driven method (matrix-method).

\subsection{Systematic uncertainties}

Several systematic uncertainties are considered :

For the backgrounds estimated by Monte Carlo simulation, uncertainties in jet and lepton efficiency, energy or momentum calibration as well as resolution are taken into account. Their effect is evaluated by varying each parameter independently within its uncertainty. The uncertainty on the luminosity is estimated from Van der Meer scans. Uncertainties on Monte Carlo background cross sections depend on the process. The trigger and reconstruction efficiency, initial and final state radiations, Parton Distribution Function, modeling of b-tagging efficiency (when b-jets are requested) and fake rates are also taken into account.

For backgrounds estimated by data driven techniques, the uncertainties on the fake signal reconstruction is estimated using variations on control region selection, and uncertainties on charge flip (for same-sign leptons analysis) is estimated by differences between three methods (tag-andprobe, direct extraction and likelihood methods).

\section{Results}

\subsection{Pair-produced heavy quarks decaying to $W q$ in the two-lepton channel}

This analysis is performed for $1.04 \mathrm{fb}^{-1}$ and the following decay chain is considered : $Q \bar{Q} \rightarrow$ $W^{+} q W^{-} \bar{q}$ where $q=d, s, b$ for up-type $Q$ and $q=u, c$ for down-type $Q$ [7].

The selection criteria are the following : two opposite sign leptons with $\left|m_{l l}-m_{Z}\right| \geq 10 \mathrm{GeV}$ and $m_{l l} \geq 15 \mathrm{GeV}$ ( $m_{l l}$ is the invariant mass of the two leptons), at least two jets, $E_{T}^{\text {miss }}$ (total missing transverse energy) $\geq 60 \mathrm{GeV}$ for $e e$ and $\mu \mu$ channel while $H_{T}\left(=\sum_{j, l} p_{T}\right) \geq 130 \mathrm{GeV}$ for $e \mu$ channel.

In order to reconstruct the mass of the heavy quark $Q$, each neutrino is assumed to be collinear with the charged lepton from the same $W$ decay as the decay products of $Q$ are boosted. The optimal values of each $|\Delta \eta(v, l)|$ and $|\Delta \phi(v, l)|$ are obtained by minimizing the mass difference between the two reconstructed heavy quarks using constraints on the reconstructed $W$ bosons, lepton directions and momenta.

Since the two reconstructed mass values $\left(m_{\text {coll }}\right)$ tend to be more correlated for signal than background, the cut $\Delta M(Q, \bar{Q}) \leq 25 \mathrm{GeV}$ is imposed to reject $t \bar{t}$.

A binned maximum likelihood ratio technique is used to fit the distributions of $m_{\text {coll }}$ shown in Figure 1(a). The limit obtained is represented on Figure 1(b). The 95\% CL observed limit is $m(Q) \geq 350 \mathrm{GeV}$ while the corresponding expected limit is $m(Q) \geq 335 \mathrm{GeV}$. 

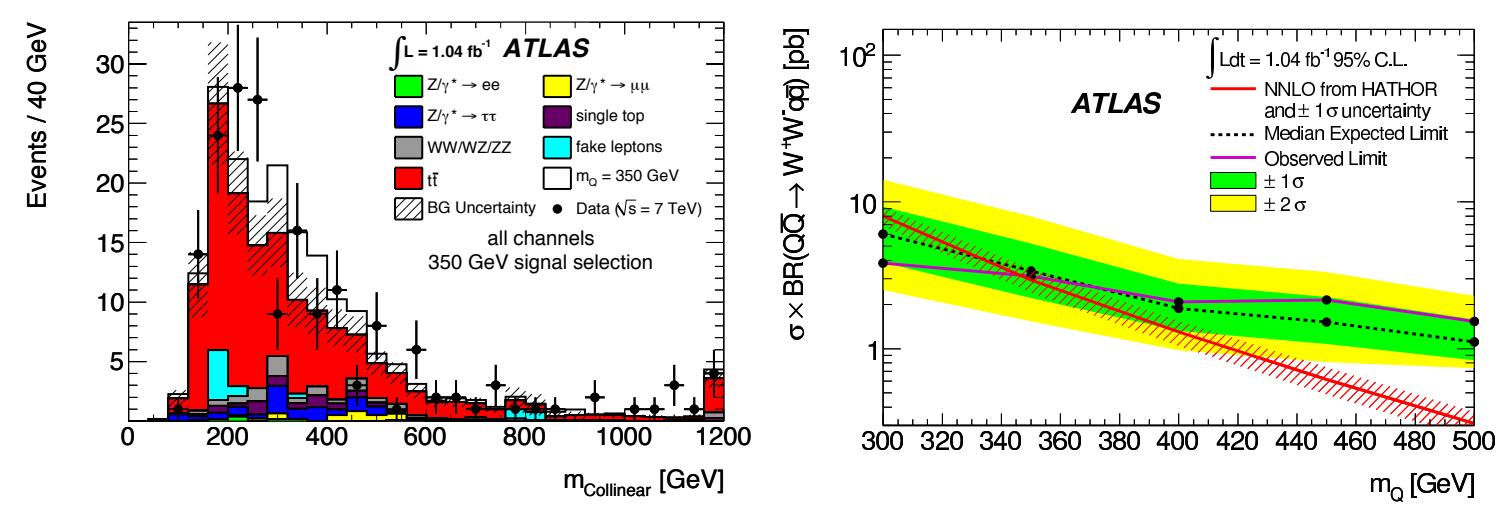

Figure 1: (left) distributions of $m_{\text {coll }}$ for the sum of each channels after applying the final selection for $m_{Q}$ $=350 \mathrm{GeV}$ and (right) mass limit obtained for the heavy quark $Q$ [7].

\subsection{Heavy top-like quarks decaying to $W b$ in the lepton plus jets final state}

The process $t^{\prime} \bar{t}^{\prime} \rightarrow W b W \bar{b}$ is searched for, using $4.7 \mathrm{fb}^{-1}$ of data [8] . Only the range $m\left(t^{\prime}\right)-$ $m\left(b^{\prime}\right)<m(W)$ is considered and events with exactly three, four or more jets are analysed separately. The signal is generated with Pythia and normalised to the approximate NNLO theoretical cross sections. Events are selected with exactly one lepton, three jets, which at least one is b-tagged, large missing transverse momentum : $E_{T}^{\text {miss }}>35(20) \mathrm{GeV}$ in the electron (muon) channel, and $E_{T}^{\text {miss }}+m_{T}>60 \mathrm{GeV}$, where $m_{T}$ is the transverse mass of the lepton and $E_{T}^{\text {miss }}$, and $W^{\prime} s$ decaying to 2 hadrons are reconstructed with the following two definitions :

- $W_{\text {had }}^{\text {typeI }}$ (single jet with $p_{T} \geq 250 \mathrm{GeV}$ and mass in $[60,110] \mathrm{GeV}$ )

- $W_{\text {had }}^{\text {typeII }}$ (dijet with $p_{T} \geq 150 \mathrm{GeV}, \Delta \mathrm{R}(\mathrm{j}, \mathrm{j})<0.8$ and $m_{j j}$ in $[60,110] \mathrm{GeV}$ )

Events are kept if there are at least 3 jets and at least one $W_{\text {had }}^{\text {typeI }}$ candidate or at least 4 jets and at least one $W_{\text {had }}^{\text {typeII }}$ candidate, $H_{T}\left(=\sum_{l, j, M E T} p_{T}\right) \geq 750 \mathrm{GeV}, p_{T}$ (leading b-jet) $\geq 160 \mathrm{GeV}$, $p_{T}($ subleading b-jet $) \geq 60 \mathrm{GeV}, \Delta \mathrm{R}(1, v)<1.4, \min \left(\Delta \mathrm{R}\left(W_{\text {had }}, b_{1,2}\right)\right)>1.4, \min \left(\Delta \mathrm{R}\left(1, b_{1,2}\right)\right)>1.4$.

The main discriminant variable is $m_{\text {reco }}$ (shown in Figure 2(a)) built from the $W_{\text {had }}$ candidate and one of the two b-jet candidates. The reconstruction of $W \rightarrow \ell v$ decay yields two solutions, and there are two possible ways to pair the b-jet candidates with the $W$ boson. Among the four possible combinations, the one yielding the smallest $\left|\Delta M\left(t^{\prime}, \bar{t}^{\prime}\right)\right|$ is chosen.

The distribution of the variable $m_{\text {reco }}$ is used in a log-likelihood ratio analysis to provide results on the $t^{\prime} \bar{t}^{\prime}$ production cross section. An expected limit of $m\left(t^{\prime}\right) \geq 638 \mathrm{GeV}$ and an observed limit of $m\left(t^{\prime}\right) \geq 656 \mathrm{GeV}$ are obtained as shown Figure 2(b).

The same analysis is used to derive exclusion limits on vector-like $t^{\prime}$ quark production, for different $m_{t}$ and as a function of $\mathrm{BR}\left(t^{\prime} \rightarrow W b\right)$ and $\mathrm{BR}\left(t^{\prime} \rightarrow H t\right) . \mathrm{BR}\left(t^{\prime} \rightarrow Z t\right)$ is fixed by $\sum B R=1$. The resulting 95\% CL exclusion limits are shown in Figure 3 for different values of $m_{t}$.

\subsection{Exotic same-sign dilepton signatures of $b^{\prime}$ quark and $T_{5 / 3}$}

The process $X \bar{X} \rightarrow W t W \bar{t}$ where $X$ stands for $b^{\prime}$ or $T_{5 / 3}$ (exotic partner of the top with charge $5 / 3$ ) with $4.7 \mathrm{fb}^{-1}$ of data is investigated in ref [9]. For the b' analysis, pair production only is taken into account while for the $T_{5 / 3}$ analysis, single production is also considered for different coupling values of $\lambda\left(T_{5 / 3}-t-W\right): \lambda \ll 1$ (equivalent to pair production only), 1 and 3 to test the value of the 

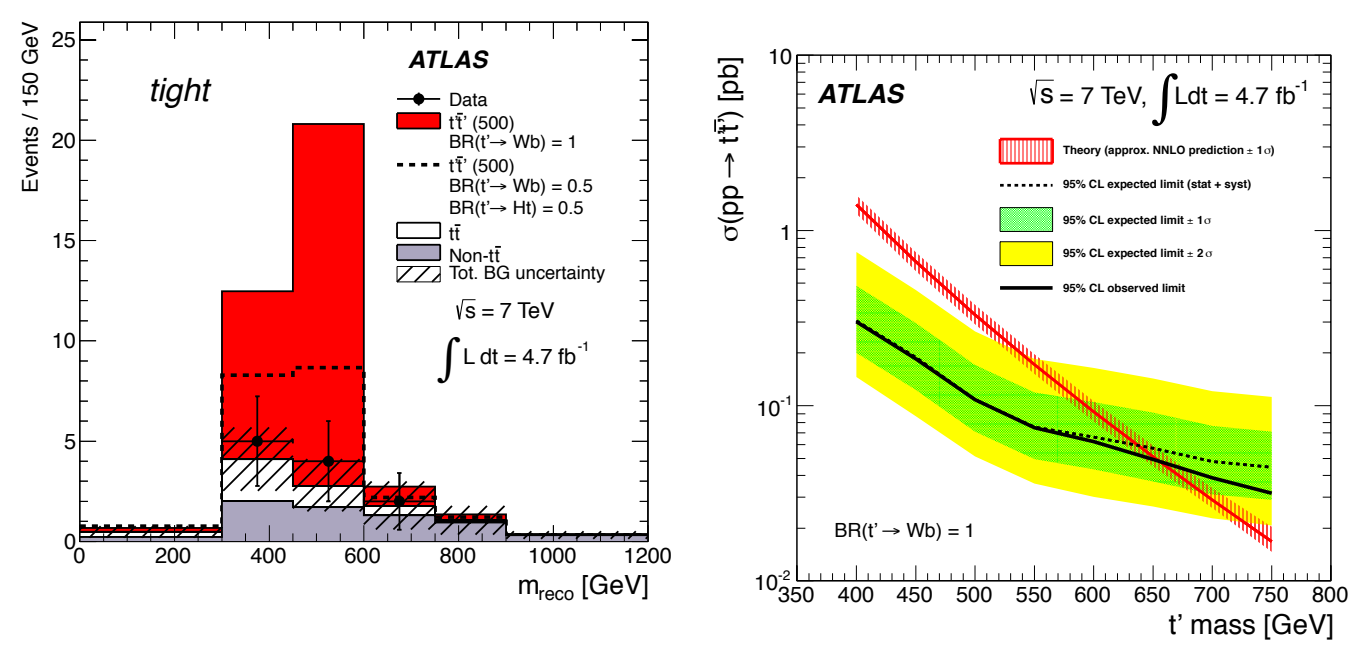

Figure 2: (left) distributions of $m_{\text {reco }}$ after cuts for $m_{t^{\prime}}=500 \mathrm{GeV}$ and (right) mass limit obtained for the $t^{\prime}$ pair cross section [8].
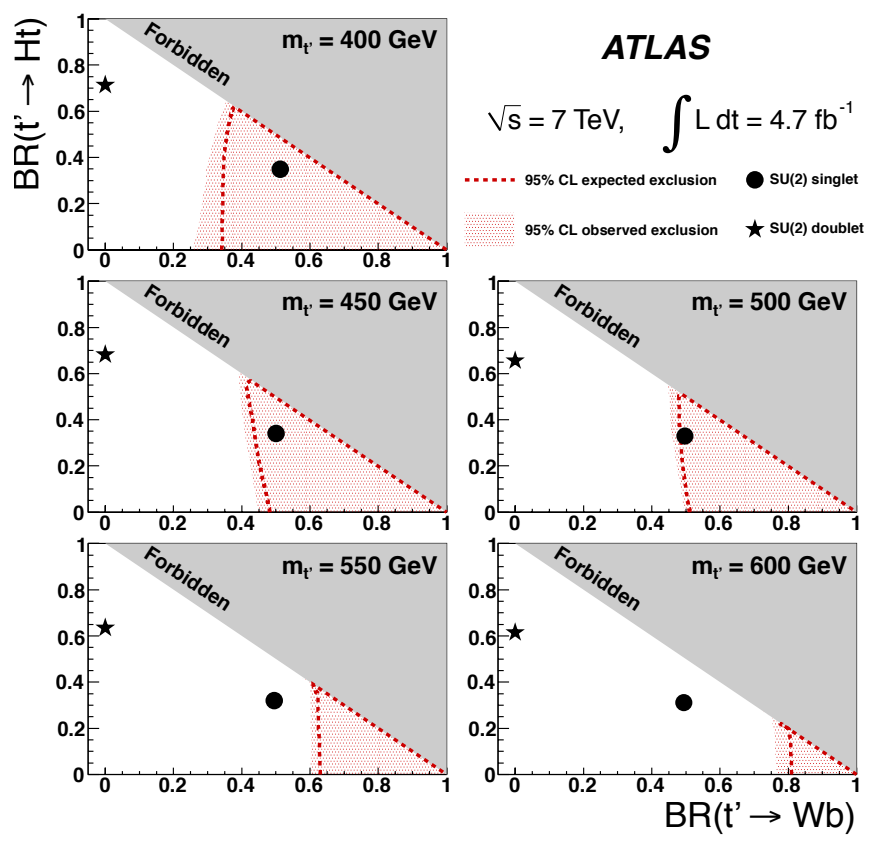

Figure 3: 95\% CL exclusion in the plane of $B R\left(t^{\prime} \rightarrow W b\right)$ versus $B R\left(t^{\prime} \rightarrow H t\right)$, for different values of $m_{t^{\prime}}$

mixing angle between SM and BSM for extra-dimensional models as it depends on the coupling value $\lambda$.

Events must have at least two same sign leptons (if multiple pairs, take the one with highest $p_{T}$ ), at least two jets (at least one is b-tagged), $E_{T}^{\text {miss }} \geq 40 \mathrm{GeV}, m_{l l} \geq 15 \mathrm{GeV},\left|m_{l l}-m_{Z}\right| \geq 10$ $\mathrm{GeV}$ (for $e e$ and $\mu \mu$ channels) and $H_{T}\left(=\sum_{j, l} p_{T}\right) \geq 550 \mathrm{GeV}$.

A cut and count method is used to extract results represented on figure 4 . The following results are obtained : $\mathrm{m}\left(b^{\prime} / T_{5 / 3}\right) \geq 670 \mathrm{GeV}$ for pair production only, $\mathrm{m}\left(T_{5 / 3}\right) \geq 680 \mathrm{GeV}$ for pair+single production with $\lambda=1$ and $\mathrm{m}\left(T_{5 / 3}\right) \geq 700 \mathrm{GeV}$ for pair+single production with $\lambda=3$. 

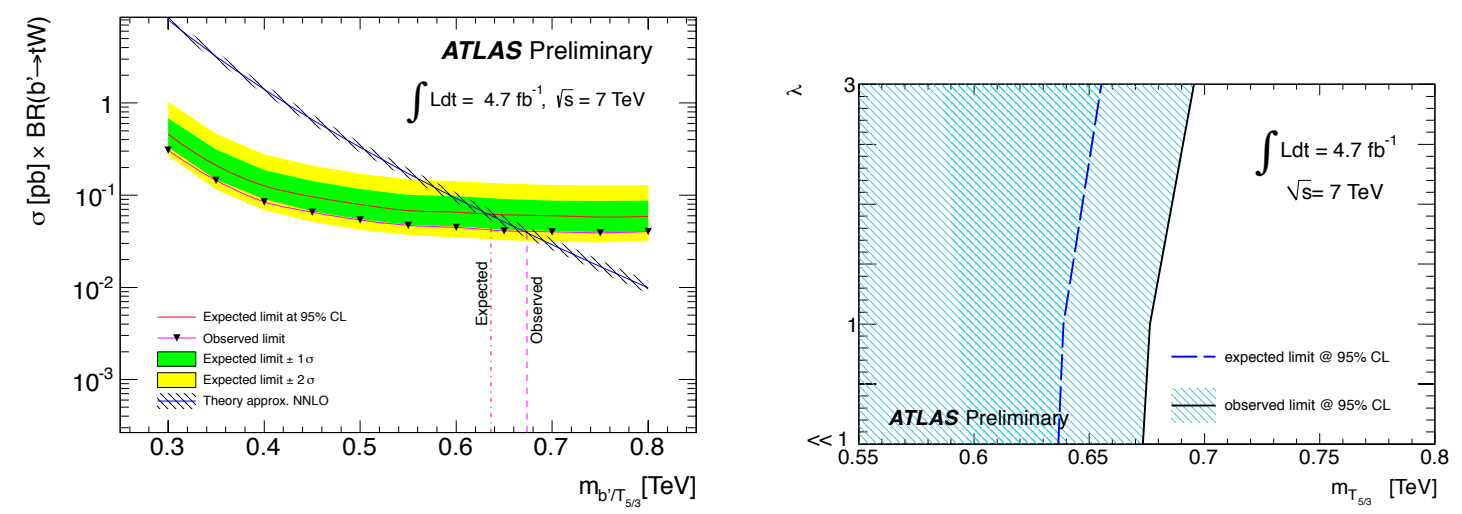

Figure 4: Observed and expected limit for the b' and $T_{5 / 3}$ for the pair production (left) and for the $T_{5 / 3}$ for the pair and single production (right) [9].

\subsection{Pair production of a new quark that decays to $Z b$}

The following decay chain is studied [10] $: b^{\prime} \bar{b}^{\prime} \rightarrow Z b+X$ where $Z \rightarrow e e$ and $X$ stands for $Z+b$ or $W+t$ with all decay modes of $Z$ and $W$ allowed. This study is performed for $1.98 \mathrm{fb}^{-1}$. The factor $\beta=2 \times B R\left(b^{\prime} \rightarrow Z b\right)-B R\left(b^{\prime} \rightarrow Z b\right)^{2}$ characterises the fraction of signal events with at least one $b^{\prime} \rightarrow Z+b$ decay. Both fourth generation quarks and Vector Like Quarks are studied : the case of a vector like singlet (VLS) mixing solely with the third SM generation is considered (assuming $m_{H} \sim 125 \mathrm{GeV}$ ) by computing $\beta$ as a function of the $b^{\prime}$ mass.

Events are selected with at least two opposite-sign leptons, $\left|m_{e e}-m_{Z}\right| \leq 5 \mathrm{GeV}$ and at least one b-tagged jet. The mass $m_{Z b}\left(=m_{b^{\prime}}\right)$ is here the discriminant variable. The $b^{\prime}$ candidate is formed from the $e^{+} e^{-}$pair and the highest $p_{T}$ b-jet. A cut $p_{T}(Z b) \geq 150 \mathrm{GeV}$ is applied to increase the signal sensitivity.

The limit is computed using a binned Poisson likelihood ratio test of the $m_{Z b}$ distribution for different $\mathrm{m}\left(b^{\prime}\right)$ hypotheses as shown in Figure 5. The cross section limit is evaluated using the CL modified frequentist approach.
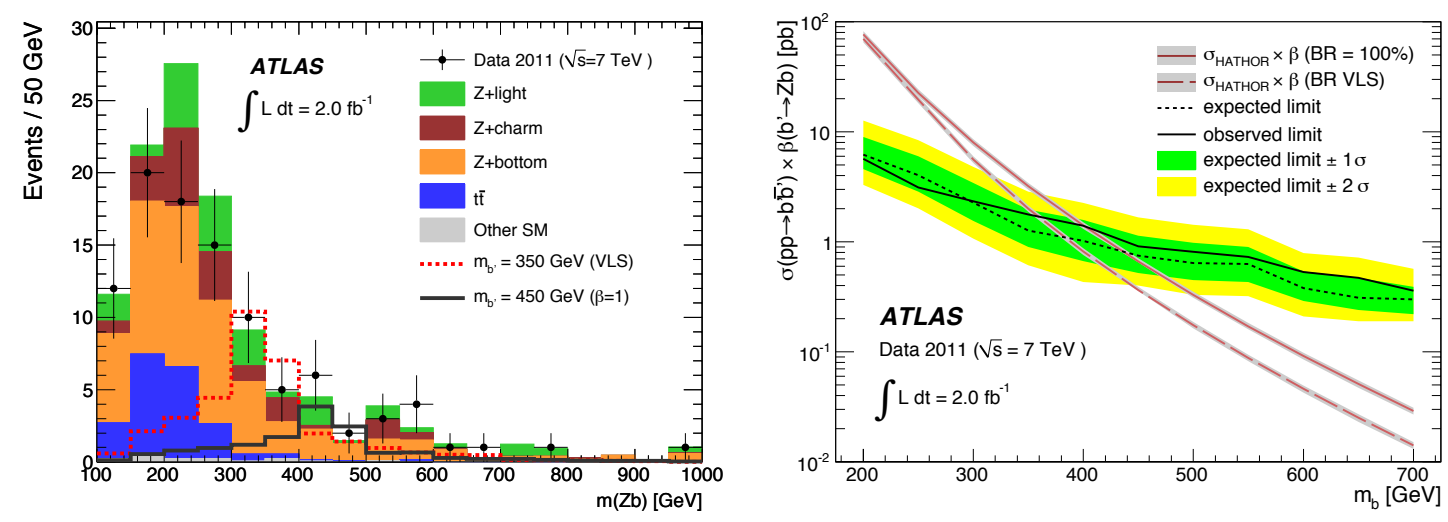

Figure 5: (left) Mass distribution of the b'candidate after cuts. (left) Observed and expected 95\% C.L. cross section limits under the assumption $\beta=1$ and assuming the VLS scenario value $\beta=0.63$ [10].

To conclude, $b^{\prime}$ quarks with masses $m_{b^{\prime}} \leq 400 \mathrm{GeV}$ decaying entirely via $b^{\prime} \rightarrow Z+b$ are 
excluded at $95 \%$ C.L and, in the case of a vector-like singlet $b^{\prime}$ mixing solely with the third SM generation, masses $m_{b^{\prime}} \leq 358 \mathrm{GeV}$ are excluded.

\subsection{Single production of VLQ coupling to light generations}

Events are analysed at $4.64 \mathrm{fb}^{-1}$ with a $W \rightarrow l v$ or $Z \rightarrow l l$ boson produced in association with at least two high $p_{T}$ jets [3].

A reference model which is compatible with electroweak precision tests has two degenerate VLQ doublets, with hypercharges $1 / 6$ and $7 / 6$, that mix only with the up quarks and are made of two quarks $U$ and $Y$ of charge $+2 / 3$, one quark $D$ of charge $-1 / 3$ and one quark $X$ of charge $+5 / 3$. The mass degeneracy ensures that corrections to SM quark couplings are not induced by the mixing. The effective coupling between light quarks and VLQs is written here $\kappa_{q Q}=\left(v / m_{Q}\right) \tilde{\kappa_{q Q}}$ where q is a light quark, $Q$ is the VLQ, v is the Higgs vacuum expectation value and $\tilde{\kappa_{q}}$ is a model-dependent parameter which could be of $\mathrm{O}(1)$.

This analysis is divided into four channels : charged current (CC) and neutral current (NC), with either $e$ or $\mu$ in the final state. The cuts are optimised independently for the CC and NC channels :

CC channel : exactly one lepton and $E_{T}^{\text {miss }} \geq 50 \mathrm{GeV}$ for the reconstruction of the $W$ (with $m_{T}(W) \geq 40 \mathrm{GeV}$ and $|\eta(W)| \leq 2.5$ ), at least two jets, $p_{T}$ (leading jet) $\geq 60 \mathrm{GeV}$, $\mid \Delta \eta(W$, leading jet $)|<2.3,| \Delta \phi(W$, leading jet $)|>2.1,| \Delta \phi\left(l, E_{T}^{\text {miss }}\right) \mid<1.3$, $\mid \Delta \eta(W$, associated jet $) \mid>1.6$ and $\mid \Delta \eta$ (leading jet, associated jet $) \mid>1.3$

NC channel : two opposite sign and same flavour leptons with $66 \mathrm{GeV} \leq m_{l l} \leq 116 \mathrm{GeV}$, at least two jets, $|\Delta \phi(l, l)|<1.5,|\Delta \eta(l, l)|<1.6, \mid \Delta \phi(Z$, leading jet $)|>2.1,| \Delta \eta(Z$, leading jet $) \mid<1.1$, $\mid \Delta \eta(Z$, associated jet $) \mid>0.9$ and $\mid \Delta \eta$ (leading jet, associated jet $) \mid>0.9$.

The background is estimated in data by fitting the reconstructed VLQ mass, calculated as the invariant mass of the reconstructed $\mathrm{W}$ or $\mathrm{Z}$ with the leading $p_{T}$ jet, and, as a cross-check, data are compared to the simulated background model. The results are consistent with a background-only hypotheses, and therefore limits are set on the production cross section $\times \mathrm{BR}$ (cf Figure 6) for the VLQ benchmark signal (Bayesian limits) as well as limit on the coupling as a function of mass (cf Figure 7) .
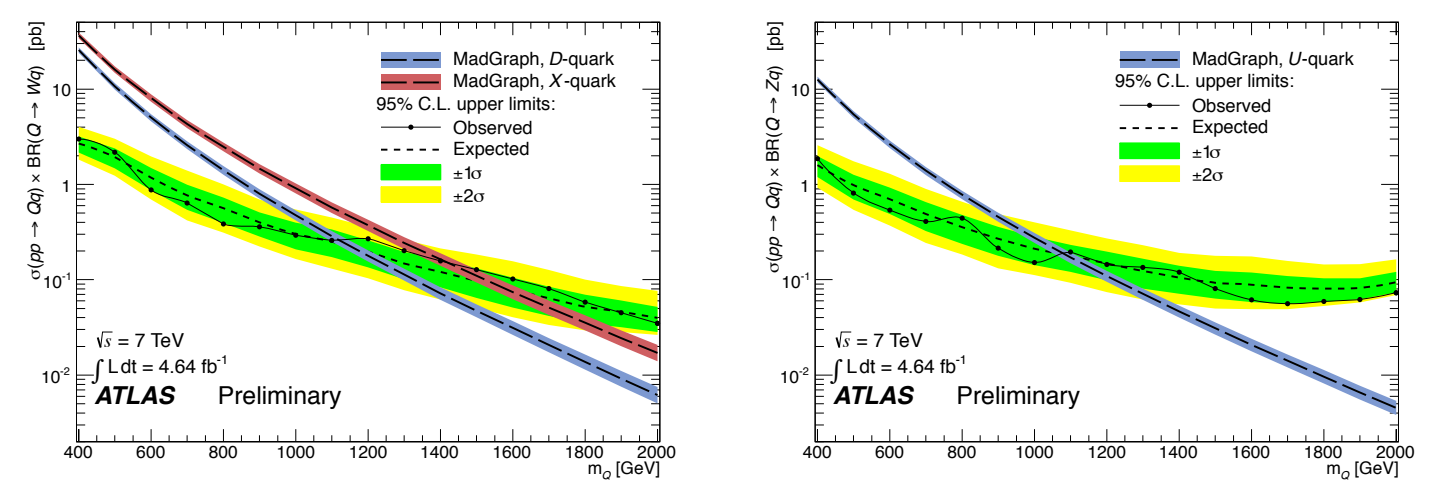

Figure 6: Observed and expected 95\% C.L. upper limits on $\sigma(p p \rightarrow q Q) \times B R(Q \rightarrow W q)$ where $Q$ is $D$ or $X($ left $)$ and $\sigma(p p \rightarrow q U) \times B R(U \rightarrow Z q)$ (right) by assuming $\tilde{\kappa_{q}}=1$ [3]. 
For the benchmark model with coupling $\tilde{\kappa_{q Q}}=1$, VLQs with charge $2 / 3$ and $-1 / 3$ with masses below $1.12 \mathrm{TeV}$ and $1.08 \mathrm{TeV}$, are excluded at $95 \%$ confidence level. For the charge +5/3 VLQ, which decays to a $W q$, masses below $1.42 \mathrm{TeV}$ are excluded.
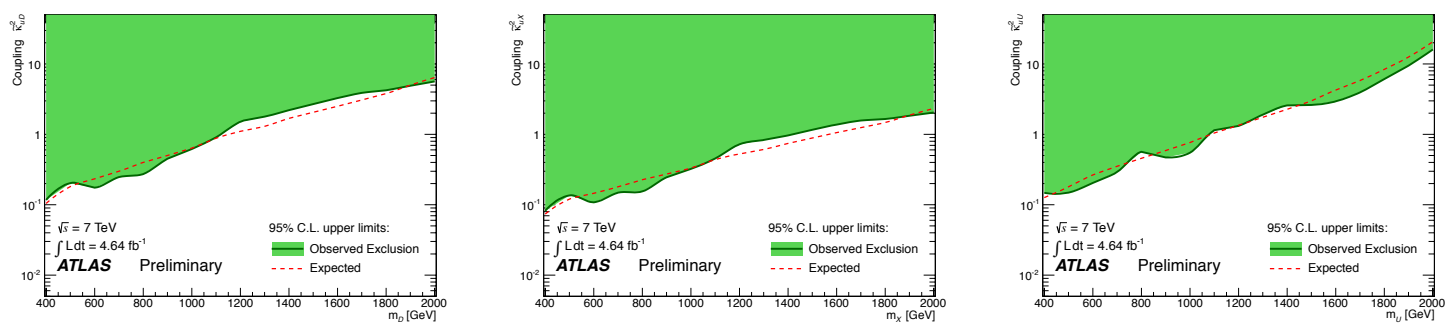

Figure 7: Observed and expected 95\% C.L. upper limits on $\tilde{\kappa_{q D}}{ }^{2}, \tilde{\kappa_{q X}}{ }^{2}$ and $\tilde{\kappa_{q U}}{ }^{2}$.

\section{Conclusion}

The fourth generation quarks model has been studied and the following results has been obtained at $95 \% \mathrm{CL}: \mathrm{m}(Q)>350 \mathrm{GeV}$ for up and down type $Q, \mathrm{~m}\left(t^{\prime}\right)>656 \mathrm{GeV}$ for $t^{\prime} \rightarrow W+b$ and $\mathrm{m}\left(b^{\prime}\right)>670 \mathrm{GeV}$ for $b^{\prime} \rightarrow W+t$. But this model is now disfavoured by $m_{H} \sim 126 \mathrm{GeV}$.

The Vector Like Quarks Model has also been studied : a VLQ interpretation of $t^{\prime}$ has been done as well as a vector-like singlet $b^{\prime}$ (with $b^{\prime} \rightarrow Z+b$ ) mixing solely with the third SM generation. Limits on VLQs with charge $5 / 3$ has been studied for different coupling value $\lambda\left(T_{5 / 3} t W\right)$ and the single production of VLQs coupling to light generations has been analysed for VLQs with charges $=-1 / 3,2 / 3$ and $5 / 3$.

\section{References}

[1] B. Holdom et al., PMC Phys. A 3, 4 (2009)

[2] ATLAS Collaboration, Phys. Rev. D86 (2012) 074014

[3] ATLAS Collaboration, ATLAS-CONF-2012-137

[4] ATLAS Collaboration, JINST 3, S08003 (2008).

[5] M.Cacciari and G.P. Salam, JHEP 0804:063,2008

[6] T. Junk, Nucl.Instrum.Meth. A434 (1999) 435-443, arXiv:hep-ex/9902006

[7] ATLAS Collaboration, Phys.Rev. D86 (2012) 012007

[8] ATLAS Collaboration, arXiv:1210.5468 [hep-ex], Accepted by Phys. Rev. Lett

[9] ATLAS Collaboration, ATLAS-CONF-2012-130

[10] ATLAS Collaboration, Phys.Rev.Lett. 109 (2012) 071801 